Service social

\title{
Les attitudes des intervenants sociaux du Québec face à l'informatisation dans leur milieu de travail
}

\section{Georgette Béliveau, Martin Poulin et Ginette Beaudoin}

Volume 36, numéro 1, 1987

L'informatique dans les services sociaux

URI : https://id.erudit.org/iderudit/706338ar

DOI : https://doi.org/10.7202/706338ar

Aller au sommaire du numéro

Éditeur(s)

École de service social de l'Université Laval

ISSN

1708-1734 (numérique)

Découvrir la revue

Citer cet article

Béliveau, G., Poulin, M. \& Beaudoin, G. (1987). Les attitudes des intervenants sociaux du Québec face à l'informatisation dans leur milieu de travail. Service social, 36(1), 13-32. https://doi.org/10.7202/706338ar
Résumé de l'article

Les intervenants sociaux ont-ils une attitude favorable ou non envers l'utilisation de l'informatique dans leur milieu de travail ? Qu'est-ce qui influence leur attitude ? Dans une recherche, les auteurs ont interrogé les employés cadres et le personnel clinique de quatre centres de services sociaux et vingt-neuf centres locaux de services communautaires du Québec, rejoignant ainsi neuf cent soixante personnes. Les attitudes ont été mesurées au moyen d'une échelle additive de type Likert. Dans cet article, les auteurs font ressortir les résultats de recherche concernant les attitudes les plus positives et les plus négatives. Ils mettent aussi en évidence les principales variables qui influencent les attitudes des intervenants. 
BÉLIVAU, Georgette, professeure à l'École de service social de l'Université Laval.

Poulın, Martin, professeur à l'École de service social de l'Université Laval.

BEAUDOIN, Ginette, professionnelle de recherche.

\section{Les attitudes des intervenants sociaux du Québec face à l'informatisation dans leur milieu de travail}

\section{Georgette Béliveau Martin Poulin Ginette Beaudoin}

L'informatique dans les services sociaux a d'abord été introduite pour supporter les systèmes d'information de gestion. Depuis quelques années, elle connaît une diffusion plus généralisée et une plus grande diversification des champs d'application. Concurremment à cette extension, l'informatique soulève de nombreuses questions et préoccupations. Notamment, en ce qui concerne les intervenants sociaux, la technologie informatique remet en cause certaines valeurs et interroge leur pratique professionnelle, particulièrement au plan de l'éthique.

Les craintes apparemment entretenues au sujet de l'utilisation des moyens informatiques dans les services sociaux sont-elles vraiment présentes chez le personnel du réseau des services? Les intervenants ont-ils' une attitude favorable ou non envers cette technologie? C'est à partir de ces interrogations que nous avons réalisé un projet de recherche qui porte sur les perceptions et les attitudes des intervenants sociaux du Québec (gestionnaires et praticiens) à l'égard des applications informatiques dans leur milieu de travail. Les personnes interrogées proviennent des centres de services sociaux (C.S.S.) et des centres locaux de services communautaires (C.L.S.C.) de quatre régions socio-sanitaires. II s'agit d'une étude de type exploratoire-descriptive. La partie de la recherche 
que nous rapportons dans cet article traite plus spécifiquement des attitudes des intervenants sociaux, sans aborder l'aspect des perceptions. ${ }^{1}$

\section{Qu'est-ce qui influence les attitudes?}

S'interroger sur ce qui influence les attitudes nous amène à élaborer le cadre de référence. Pour cela, nous nous sommes principalement inspirés de la théorie du comportement organisationnel, laquelle permet d'accéder à une vision globale et intégrée d'un ensemble de facteurs mutuellement interreliés parmi lesquels les facteurs humains jouent un rôle déterminant. Tout en considérant les facteurs liés aux organisations, cette théorie s'intéresse à l'individu ; par conséquent, elle nous permet de définir et d'expliquer les concepts de perception et d'attitude. Cette approche s'inspire aussi des théories du management et des organisations, d'où sa vision intégrée des dimensions organisationnelles reliées, entre autres, à la structure, à la technologie, aux facteurs de groupe et de personnalité associés aux besoins, attentes et attitudes. Pour les fins de notre étude, nous avons retenu le schéma de référence suivant :

\section{GRAPHIQUE 1}

\section{Cadre de référence}

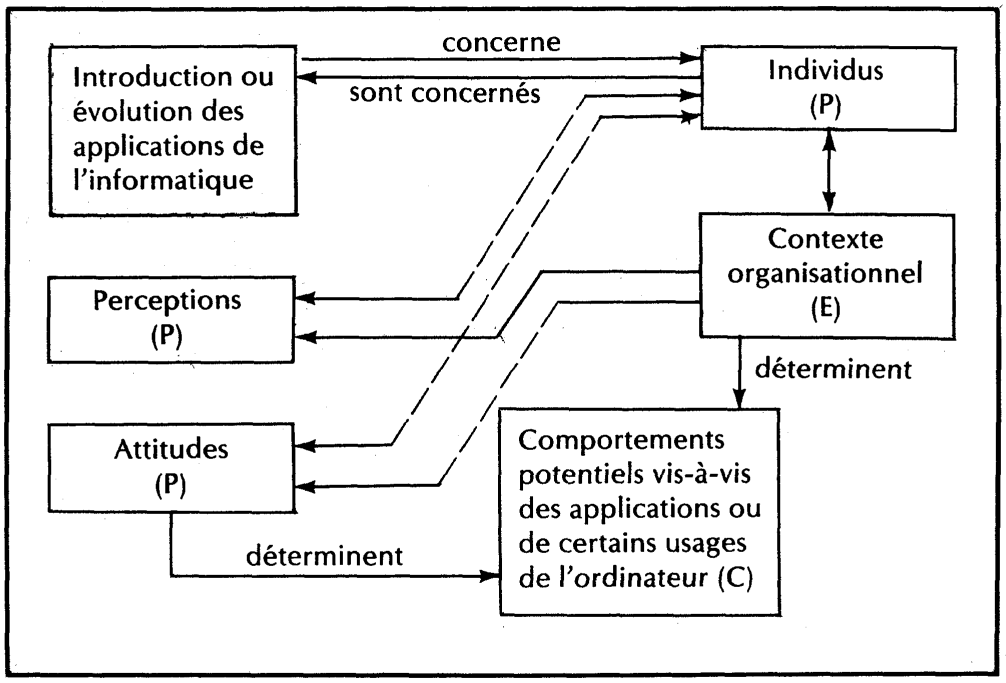


Dans ce schéma, les perceptions et les attitudes font référence aux facteurs de personnalité $(P)$ et se rapportent aux individus : ceux-ci se situent dans un contexte organisationnel qui fait référence à l'environnement (E). Ce qu'on appelle "comportements potentiels " face aux utilisations de l'ordinateur correspond aux comportements de l'individu (C). Donc, dans notre étude, ces comportements potentiels vis-à-vis les applications de l'ordinateur sont déterminés par des facteurs reliés à l'individu et au contexte organisationnel.

Par ailleurs, les recherches antérieures nous ont permis d'identifier un certain nombre de facteurs influençant les attitudes. Parmi ceux-ci, nous pouvons faire une distinction entre ceux reliés aux individus (position hiérarchique et degré de contact avec l'ordinateur) et ceux reliés au contexte organisationnel (taille de l'organisation, durée d'informatisation et type d'établissement). Le graphique 1 illustre la relation déterminante entre ces deux types de facteurs et les perceptions et attitudes.

Ce cadre de référence nous permet de distinguer deux groupes d'hypothèses; les premières, liées aux individus, examinent dans quelle mesure certaines caractéristiques personnelles peuvent influencer les attitudes, alors que celles ayant trait au contexte organisationnel portent sur l'effet qu'ont certains facteurs liés à l'organisation sur les perceptions et les attitudes. Nous avons formulé comme suit les hypothèses reliées aux attitudes:

1. Relativement aux individus, les attitudes des intervenants sociaux à l'égard de l'informatique varient selon : leur position hiérarchique et leur degré de contact avec l'informatique.

2. En ce qui a trait au contexte organisationnel, elles varient selon: la taille de l'organisation, la durée d'informatisation et le type d'établissement.

Le graphique 2 permet de visualiser les différentes variables (dépendante et indépendantes) en cause dans nos hypothèses, ainsi que les relations entre elles. 


\section{GRAPHIQUE 2}

\section{Relations entre variables indépendantes et variable dépendante}

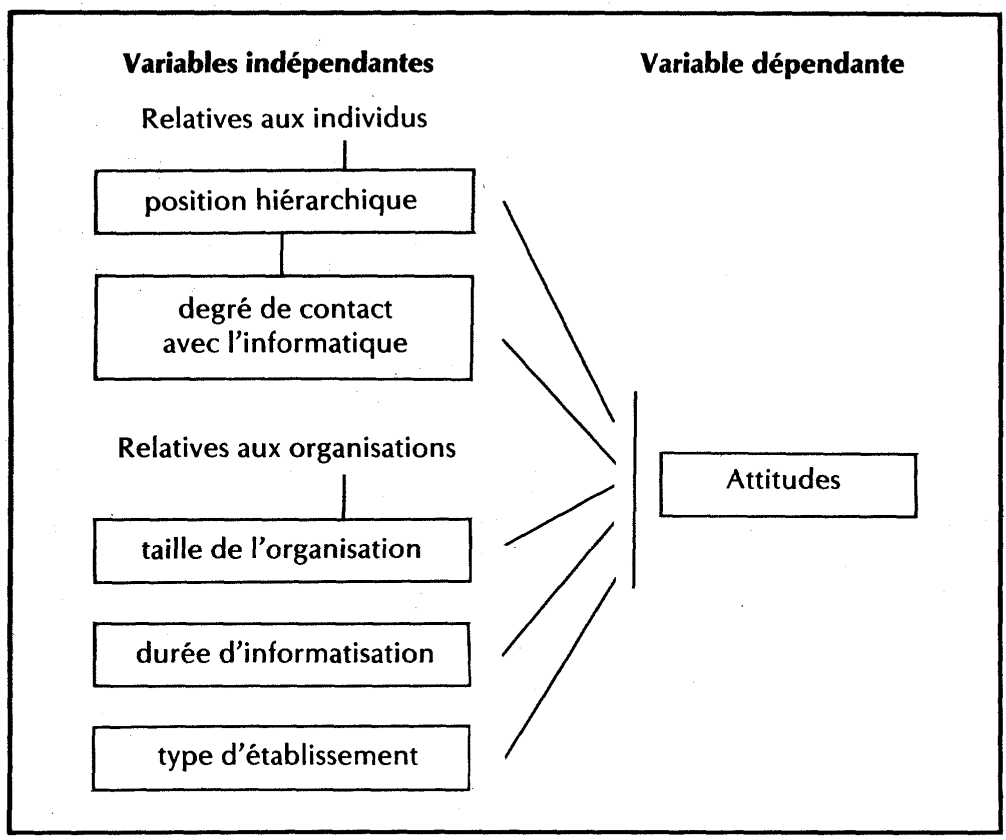

Nous avons également retenu certaines variables de contrôle : le sexe, l'âge, la formation, la discipline d'études, les années d'expérience et le secteur d'activités. Elles sont connues comme pouvant avoir un effet sur la formation des attitudes.

\section{Comment avons-nous mesuré les attitudes?}

L'attitude dispose à l'action et exerce une influence immédiate sur le comportement. La définition retenue le plus couramment chez les spécialistes des sciences humaines est la suivante : "Une attitude, c'est une prédisposition à réagir d'une façon systématique favorable ou défavorable face à certains aspects du monde environnant " (Bergeron et al., 1979, p. 84). Par ailleurs, connaître l'attitude d'un individu envers 
un phénomène donné, c'est aussi pouvoir prédire, d'une certaine façon, comment il va réagir. L'attitude fait appel à la connaissance ou à l'idée qu'a un individu d'un objet, et fait également appel aux sentiments qui surgissent lorsqu'il pense à cet objet; nous la définissons donc comme la réaction qu'a une personne par rapport à un objet donné.

Afin de mesurer quantitativement l'attitude à l'égard de l'informatique, nous avons construit et validé une échelle de vingt-trois énoncés, couvrant diverses dimensions caractérisant l'informatique dans les services sociaux. À chaque énoncé, l'intervenant devait situer sa réponse sur un continuum gradué, allant de 1 (fortement en désaccord) à 5 (fortement en accord). Les réponses à l'ensemble des énoncés permettaient d'obtenir une mesure des attitudes.

Certaines caractéristiques propres aux individus peuvent influencer les attitudes à l'égard de l'informatique. Les résultats des recherches (Lynch, 1985 ; Pinkerton et Raffoul, 1984) nous ont montré que, dépendamment de son degré de contact avec l'informatique ou de sa position hiérarchique, l'intervenant démontrait des attitudes différentes. Voici comment ces deux variables ont été opérationnalisées. Pour la position hiérarchique, nous avons regroupé les intervenants selon leur appartenance aux trois groupes suivants : les praticiens, les cadres intermédiaires et les cadres supérieurs. Le degré de contact avec l'informatique a été établi à partir d'un indice énumératif, qui provient des réponses données à six questions portant sur le contact avec l'informatique à la maison, au travail et en dehors du travail. Le degré de contact est proportionnel au nombre de réponses positives indiquant une forme de contact avec l'informatique. Il se mesure par la somme des scores obtenus à l'ensemble des questions, un résultat élevé indiquant un bon degré de contact avec l'informatique.

Nous avons retenu, relativement au contexte organisationnel, les variables suivantes : la taille de l'organisation, la durée d'informatisation et le type d'établissement. Chacune de ces variables a été opérationnalisée de la façon suivante. La taille de l'organisation (C.S.S. ou C.L.S.C.) a été mesurée par le nombre d'employés travaillant à temps complet et à temps partiel dans l'établissement. Nous avons formé trois catégories: moins de 100 employés, entre 100 et 200 employés, et plus de 200 employés. Par la durée d'informatisation, nous entendons le nombre d'années depuis lesquelles l'informatique est utilisée. En nous basant sur les propos de Gummer (1986), nous avons distingué les établissements où l'informatique est utilisée depuis cinq ans et moins, et depuis six ans et plus. Comme troisième variable organisationnelle, nous avons retenu le type d'établissement, soit C.S.S. ou C.L.S.C. 
Notre recherche vise les intervenants sociaux employés comme personnel cadre ou personnel clinique dans les services sociaux externes, soit les C.S.S. et les C.L.S.C. En 1985, le Ministère de la santé et des services sociaux (M.S.S.S.) procédait au parachèvement du réseau des C.L.S.C. du Québec. Parmi les cent quarante-sept existant alors, certains n'en étaient qu'à l'étape d'implantation. Pour cette raison, nous n'avons considéré que ceux fondés avant le premier janvier 1984. À cette période, le réseau comprenait cent dix C.L.S.C. répondant à cette caractéristique.

Pour le choix des régions socio-sanitaires, nous avons sélectionné trois régions et une sous-région qui se distinguent par la densité de leur population. Parmi les sept régions administratives du Québec dont la densité est inférieure à $10 \mathrm{hab} . / \mathrm{km}^{2}$, nous avons retenu le Saguenay/ Lac-Saint-Jean/Chibougamau (région 02) et l'Outaouais (région 07); des deux régions de densité moyenne (20 à $\left.30 \mathrm{hab} . / \mathrm{km}^{2}\right)$, l'Estrie (région 05) a été choisie ; finalement, de la région métropolitaine de Montréal dont la densité de population est de $89 \mathrm{hab}$. et plus $/ \mathrm{km}^{2}$, et qui se subdivise en trois sous-régions, nous avons retenu la Montérégie (sud de Montréal, région 06C). Le territoire couvert par l'étude représente 29,4\% de l'ensemble de la population du Québec.

Afin d'assurer l'homogénéité du groupe visé par l'étude, nous avons défini comme « intervenant social » toute personne cuvrant dans un C.S.S. ou un C.L.S.C., comme gestionnaire ou praticien, et répondant à au moins un des trois critères suivants : avoir une formation universitaire ou collégiale en sciences humaines (sciences sociales, sociologie, administration, droit, éducation, psychologie, criminologie, etc.), être membre de la Corporation professionnelle des travailleurs sociaux ou de l'Association des conseillers sociaux, être classifié " agent de relations humaines " par l'établissement.

Nous avons choisi le questionnaire comme instrument principal de collecte des données. Il comporte vingt-quatre questions regroupées en trois parties. Les premières portent sur différentes caractéristiques personnelles du répondant: sexe, âge, diplôme obtenu, discipline d'études, années d'expérience, statut d'emploi (à temps complet ou à temps partiel), fonction occupée, position hiérarchique, secteur d'activités. La deuxième partie du questionnaire a trait au degré de contact avec l'informatique ; la dernière comprend huit questions sur les perceptions et une autre sur les attitudes à l'égard de l'informatique. Les résultats relatifs à cette dernière partie, laquelle comporte vingt-trois énoncés, font l'objet du présent article.

Des 1397 questionnaires acheminés aux personnes éligibles à notre recherche, 960 ont été remplis et retournés entre le 15 octobre et le 
15 décembre 1986. Ceci représente un taux de réponse de $68.7 \%$ et touche quatre C.S.S. et vingt-neuf C.L.S.C. répartis dans les régions socio-sanitaires retenues (voir tableau 1).

\section{TABLEAU 1}

Envois et taux de retours du questionnaire par région.

\begin{tabular}{|c|c|c|c|c|c|}
\hline \multirow{3}{*}{$\begin{array}{l}\text { Régions } \\
\text { socio-sanitaires } \\
\begin{array}{l}\text { Saguenay/Lac Saint-Jean/ } \\
\text { Chibougamau (02) }\end{array}\end{array}$} & \multirow{3}{*}{$\begin{array}{c}\begin{array}{c}\text { C.S.S. } \\
(\mathbf{N})\end{array} \\
1\end{array}$} & \multirow{2}{*}{$\begin{array}{c}\text { C.L.S.C. } \\
\text { (N) }\end{array}$} & \multirow{2}{*}{$\begin{array}{l}\text { Envois } \\
(\mathbf{N})\end{array}$} & \multicolumn{2}{|c|}{ Retours } \\
\hline & & & & (N) & $\%$ \\
\hline & & 5 & 223 & 176 & $79.1 \%$ \\
\hline Estrie (05) & 1 & 4 & 217 & 153 & $69.7 \%$ \\
\hline $\begin{array}{l}\text { Sud de Montréal } \\
\text { Montérégie }(06 \mathrm{C})\end{array}$ & 1 & 12 & 693 & 435 & $62.6 \%$ \\
\hline Outaouais (07) & 1 & 8 & 264 & 196 & $73.9 \%$ \\
\hline Total & $\begin{array}{c}4 / 14 \\
28.5 \%\end{array}$ & $\begin{array}{l}29 / 110 \\
26.3 \%\end{array}$ & 1397 & 960 & $68.7 \%$ \\
\hline
\end{tabular}

\section{Profil des répondants et des établissements}

Les données qui suivent permettent de mieux connaître le profil général des intervenants sociaux, leur degré de contact avec l'informatique, et certaines caractéristiques de leurs établissements.

Les résultats concernant les caractéristiques personnelles des répondants révèlent un nombre plus important de femmes (59.2\%) que d'hommes (40.8\%), que l'âge varie de 30 à 39 ans dans plus de la moitié des cas $(51.8 \%)$, que l'on est détenteur à $51.5 \%$ d'un baccalauréat, à $84.7 \%$ d'un diplôme universitaire et que $61.2 \%$ des répondants possèdent une formation collégiale ou universitaire en service social. La majorité (77.4\%) des intervenants sont des praticiens.

Pour ce qui est des établissements, $57.5 \%$ emploient moins de cent personnes et $70 \%$ possèdent un système informatique depuis six ans et plus. Concernant le degré de contact avec l'informatique, nous pouvons retenir que les praticiens possèdent un degré de contact visiblement plus faible que celui des cadres intermédiaires et supérieurs, et que les femmes ont un degré de contact plus faible que les hommes. 


\section{TABLEAU 2}

\section{Énoncés pour lesquels on a le plus une attitude positive, négative, partagée ou incertaine.}

\begin{tabular}{|c|c|c|}
\hline Attitude positive & Attitude négative & $\begin{array}{l}\text { Attitude partagée } \\
\text { ou incertaine }\end{array}$ \\
\hline $\begin{array}{l}\text { - C'est gaspiller que d'investir temps et } \\
\text { argent dans la formation du personnel } \\
\text { en informatique } \\
\text { (en désaccord : } 75.5 \%)^{*}\end{array}$ & $\begin{array}{l}\text { - L'informatique néglige l'aspect qualitatif } \\
\text { du travail } \\
\text { (en accord : } 55.3 \% \text { ) }\end{array}$ & $\begin{array}{l}\text { - L'informatique favorise l'équité dans } \\
\text { la distribution des ressources ou des } \\
\text { services } \\
\text { (incertains : } 47.8 \% \text { ) }\end{array}$ \\
\hline $\begin{array}{l}\text { - L'informatique et les ordinateurs sont } \\
\text { à mon avis indispensables } \\
\text { (en accord : } 63.4 \%)\end{array}$ & $\begin{array}{l}\text { - L'autonomie des praticiens dans leur } \\
\text { intervention avec la clientèle est } \\
\text { menacée par l'informatique } \\
\text { (en accord : } 49.3 \% \text { ) }\end{array}$ & $\begin{array}{l}\text { - Avec l'informatique, les renseigne- } \\
\text { ments sont utilisés de façon abusive } \\
\text { (incertains : } 40.9 \% \text { ) }\end{array}$ \\
\hline $\begin{array}{l}\text { - L'informatique fournit des informa- } \\
\text { tions précises et exactes } \\
\text { (en accord : } 60.2 \% \text { ) }\end{array}$ & $\begin{array}{c}\text { - L'informatique impose des catégorisa- } \\
\text { tions qui ne collent pas à la pratique } \\
\text { (en accord: } 47.5 \% \text { ) }\end{array}$ & $\begin{array}{l}\text { - L'informatique aide à la qualité des } \\
\text { services à la clientèle } \\
\text { (incertains : } 37.5 \% \text { ) }\end{array}$ \\
\hline
\end{tabular}




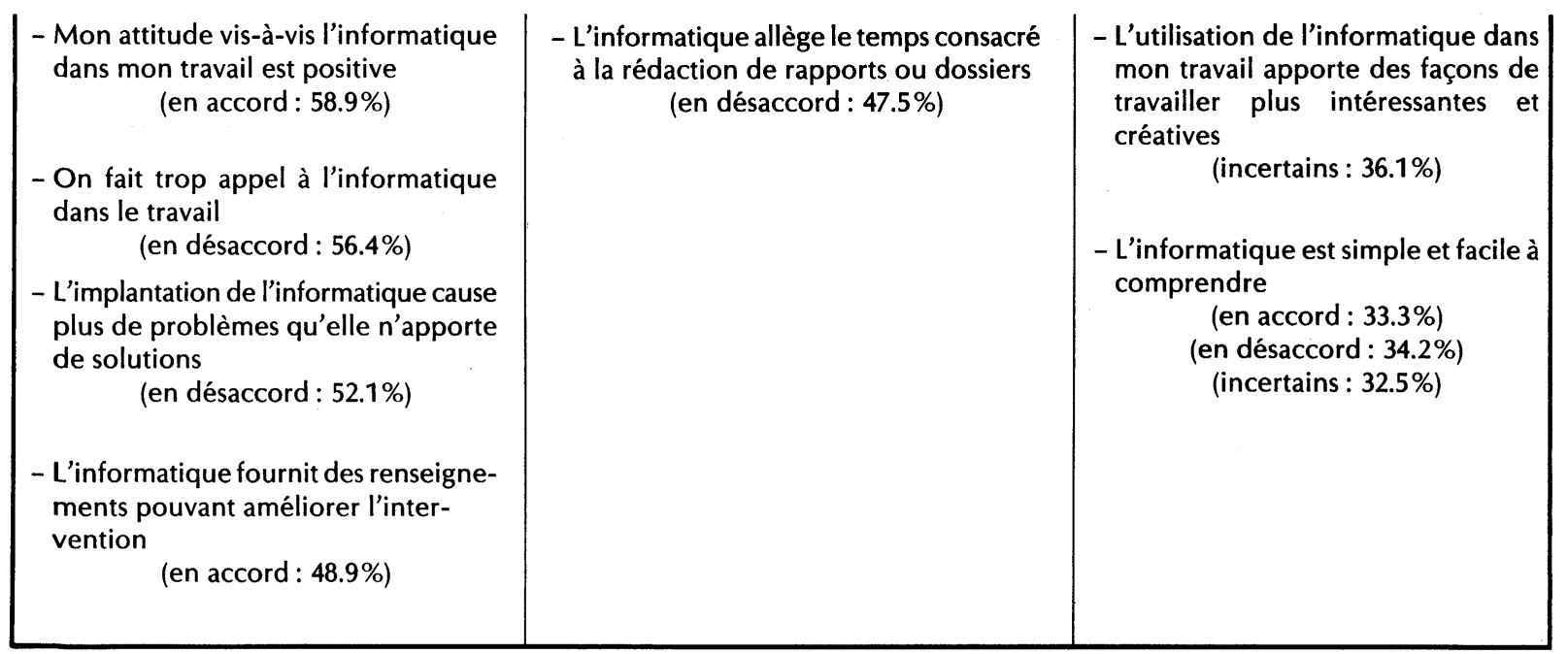

* Les pourcentages en accord ont été obtenus par la sommation des fréquences "fortement d'accord " et "d'accord ". Les pourcentages en désaccord résultent de la sommation des fréquences "fortement en désaccord " et "en désaccord ". 


\section{Attitudes : des plus positives aux plus négatives}

En ce qui concerne les attitudes, nous avons retenu les énoncés qui nous semblaient les plus significatifs ( 16 sur 23 ). Le tableau 2 fait ressortir les énoncés de l'échelle d'attitudes pour lesquels les répondants ont exprimé des attitudes positives, négatives ou incertaines.

C'est à l'égard de l'énoncé favorisant la formation du personnel à l'informatique que les intervenants sociaux ont répondu le plus posivitement $(75.5 \%)$. Nous relevons dans le tableau que plus de la moitié des répondants voient négativement le rôle de l'informatique à l'égard de l'aspect qualitatif du travail ( $55.3 \%)$, et près de la moitié craignent pour l'autonomie des praticiens dans leur intervention (49.3\%). Pour ceux ayant une attitude incertaine, c'est à l'énoncé portant sur l'équité dans la distribution des ressources ou des services qu'on les retrouve en plus grand nombre ( $47.8 \%$ ), suivi de l'énoncé stipulant que les renseignements sont utilisés de façon abusive (40.9\%). Dans l'ensemble des 23 énoncés, seulement 2 ont moins de $20 \%$ d'incertains et 10 en ont plus de $30 \%$. Ces résultats reflètent un niveau important d'ambivalence dans les attitudes des répondants.

\section{Facteurs influençant les attitudes}

Après avoir analysé les relations entre les attitudes et chacune des variables indépendantes, à l'aide du test $X^{2}$, il s'est avéré que la position hiérarchique, le degré de contact avec l'informatique et le type d'établissement étaient en relation significative avec les attitudes. Par contre, la taille de l'établissement et la durée d'informatisation ne créaient pas de différences significatives.

\section{La position hiérarchique}

La position hiérarchique comportait trois catégories: praticiens, cadres intermédiaires et cadres supérieurs. À la lecture du tableau 3, on peut constater une relation très significative (seuil de signification: .00001) entre le niveau hiérarchique et les attitudes. En examinant les résultats sous l'angle du rapport attitude/position hiérarchique, nous pouvons observer que plus la position hiérarchique est élevée, plus on a une proportion importante de personnes ayant une attitude positive envers l'informatique. En effet, $82.5 \%$ des gestionnaires de niveau supérieur ont une attitude plutôt positive, comparativement à $63.0 \%$ des gestionnaires de niveau intermédiaire et $23.7 \%$ des praticiens. 


\section{TABLEAU 3}

Attitudes selon la position hiérarchique.

\begin{tabular}{|c|c|c|c|c|}
\hline $\begin{array}{l}\text { Attitude } \\
\text { Position } \\
\text { hiérarchique }\end{array}$ & $\begin{array}{l}\text { Plutôt } \\
\text { négative } \\
\% \quad(\mathbf{N})\end{array}$ & $\begin{array}{l}\text { Incertaine } \\
\qquad \begin{array}{l}\text { (N) }\end{array}\end{array}$ & $\begin{array}{l}\text { Plutôt } \\
\text { positive } \\
\% \quad(\mathbf{N})\end{array}$ & $\begin{array}{l}\text { TOTAL } \\
\% \quad(\mathbf{N})\end{array}$ \\
\hline Praticiens & $\begin{array}{l}41.2(279) \\
31.8\end{array}$ & $\begin{array}{l}35.1(238) \\
27.2\end{array}$ & $\begin{array}{l}23.7(161) \\
18.4\end{array}$ & $\begin{aligned} 100.0 \\
77.4\end{aligned}$ \\
\hline $\begin{array}{l}\text { Cadres } \\
\text { intermédiaires }\end{array}$ & $\begin{array}{ll}7.4 & (10) \\
1.1 & \end{array}$ & $\begin{aligned} 29.6 & (40) \\
4.6 & \end{aligned}$ & $\begin{array}{rr}63.0 & (85) \\
9.7 & \end{array}$ & $\begin{array}{c}100.0(135) \\
15.4\end{array}$ \\
\hline $\begin{array}{l}\text { Cadres } \\
\text { supérieurs }\end{array}$ & $\begin{array}{ll}0.0 & (0) \\
0.0 & \end{array}$ & $\begin{array}{rr}17.5 & (11) \\
1.3 & \end{array}$ & $\begin{array}{rr}82.5 & (52) \\
5.9 & \end{array}$ & $\begin{array}{rr}100.0 & (63) \\
7.2 & \end{array}$ \\
\hline TOTAL & 32.9 (289) & 33.1 (289) & $34.0(298)$ & $100.0(876)$ \\
\hline $\begin{array}{c}X^{2}=165.11281^{*} \\
C=0.39\end{array}$ & \multicolumn{4}{|c|}{$\begin{array}{c}P=0.00000(<.00001 \text { significatif }) \\
D L=4\end{array}$} \\
\hline
\end{tabular}

* $\mathrm{X}^{2}$ : test permettant de déterminer s'il y a relation entre deux variables, compte tenu du degré de liberté.

$P$ : test de signification; indique la probabilité que la valeur du $X^{2}$ soit produite au hasard de la fluctuation de l'échantillonnage.

C : coefficient de contingence; si la relation est significative, nous pouvons estimer son intensité à l'aide de ce coefficient.

DL : degré de liberté, c'est-à-dire produit du nombre de rangées moins un et du nombre de colonnes moins un.

\section{Le degré de contact avec l'informatique}

Nous pouvons observer, au tableau 4, que la proportion d'intervenants ayant une attitude positive est considérablement plus importante chez ceux possédant un degré de contact élevé (73\%) qu'un contact moyen $(35.4 \%)$ ou faible $(23.2 \%)$. Les écarts sont aussi considérables lorsqu'on examine la proportion d'intervenants ayant une attitude négative; dans ce cas, les sujets possédant un degré de contact faible sont les plus nombreux (46.4\%) à réagir de cette façon. Tout porte à croire que, plus les intervenants possèdent un degré de contact élevé, plus ils sont favorables à l'informatique. 


\section{TABLEAU 4}

\section{Attitudes selon le degré de contact avec l'informatique.}

\begin{tabular}{|c|c|c|c|c|}
\hline Contact & $\begin{array}{l}\text { Plutôt } \\
\text { négative } \\
\% \quad(N)\end{array}$ & $\begin{array}{l}\text { Incertaine } \\
\% \quad(\mathbf{N})\end{array}$ & $\begin{array}{l}\text { Plutôt } \\
\text { positive } \\
\% \quad(\mathbf{N})\end{array}$ & $\begin{array}{l}\text { TOTAL } \\
\%(\mathbf{N})\end{array}$ \\
\hline $\begin{array}{l}\text { Faible } \\
17.0\end{array}$ & $\begin{array}{l}46.4(148) \\
11.1\end{array}$ & $\begin{aligned} 30.4 & (97) \\
8.5 & \end{aligned}$ & $\begin{array}{ll}23.2 & (74) \\
36.7 & \end{array}$ & 100.0 \\
\hline $\begin{array}{l}\text { Moyen } \\
15.8\end{array}$ & $\begin{array}{l}28.2(138) \\
20.4\end{array}$ & $\begin{array}{l}36.4(178) \\
19.9\end{array}$ & $\begin{array}{l}35.4(173) \\
56.1\end{array}$ & 100.0 \\
\hline $\begin{array}{c}\text { Élevé } \\
0.2\end{array}$ & $\begin{array}{ll}3.2 & (2) \\
1.7\end{array}$ & $\begin{array}{rr}23.8 & (15) \\
5.3 & \end{array}$ & $\begin{aligned} 73.0 & (46) \\
7.2 & \end{aligned}$ & 100.0 \\
\hline TOTAL & 33.0 (288) & $33.2(290)$ & 33.7 (293) & $100.0(871)$ \\
\hline $\begin{array}{c}X^{2}=81.37050 \\
C=0.29\end{array}$ & \multicolumn{4}{|c|}{$\begin{array}{c}P=0.0000(<.0001 \text { significatif }) \\
D L=4\end{array}$} \\
\hline
\end{tabular}

Le type d'établissement

Au tableau 5, les données nous indiquent que les attitudes diffèrent de façon significative selon que les intervenants ouvrent dans un C.S.S. ou un C.L.S.C. Nous pouvons observer que les intervenants provenant des C.L.S.C. sont plus nombreux à avoir une attitude négative (39.4\%) que ceux des C.S.S. (27.8\%). La répartition des «incertains » et de ceux qui affichent une attitude "plutôt positive " est quasi égale pour chaque type d'établissement : $35.9 \%$ et $36.3 \%$ dans les C.S.S., $29.5 \%$ et $31 \%$ dans les C.L.S.C.

Trois des cinq variables indépendantes se trouvent donc en relation significative avec les attitudes: la position hiérarchique, le degré de contact avec l'informatique et type d'établissement. Par contre, le fait d'appartenir à une organisation de petite, moyenne ou grande taille, informatisée depuis 5 ans et moins ou 6 ans et plus, ne fait pas varier les attitudes de façon significative.

Après avoir examiné les relations entre les attitudes et chacune des variables de contrôle, il s'est avéré que le sexe, l'âge, la discipline d'études et le secteur d'activités étaient en relation significative avec les attitudes. Seules les variables ayant trait au diplôme et aux années de travail ne l'étaient pas. 
TABLEAU 5

Attitudes selon le type d'établissement.

\begin{tabular}{|c|c|c|c|c|}
\hline Établissement & $\begin{array}{l}\text { Plutôt } \\
\text { négative } \\
\% \quad \text { (N) }\end{array}$ & $\begin{array}{l}\text { Incertaine } \\
\begin{array}{c}\% \quad(\mathbf{N})\end{array}\end{array}$ & $\begin{array}{l}\text { Plutôt } \\
\text { positive } \\
\% \quad \text { (N) }\end{array}$ & $\begin{array}{l}\text { TOTAL } \\
\% \quad(\mathbf{N})\end{array}$ \\
\hline C.S.S. & $\begin{array}{l}27.8(136) \\
15.4\end{array}$ & $\begin{array}{l}35.9(176) \\
19.9\end{array}$ & $\begin{array}{l}36.3(178) \\
20.2\end{array}$ & $\begin{array}{c}100.0(490) \\
55.5\end{array}$ \\
\hline C.L.S.C. & $\begin{array}{l}39.4(155) \\
17.6\end{array}$ & $\begin{array}{l}29.5(116) \\
13.1\end{array}$ & $\begin{array}{l}31.0(122) \\
13.8\end{array}$ & $\begin{array}{c}100.0(393) \\
44.5\end{array}$ \\
\hline TOTAL & 33.0 (291) & $33.1(292)$ & $34.0(300)$ & $100.0(883)$ \\
\hline $\begin{array}{c}X^{2}=13.53020 \\
C=0.12\end{array}$ & \multicolumn{4}{|c|}{$\begin{array}{c}P=0.0012(<.01 \text { significatif }) \\
D L=2\end{array}$} \\
\hline
\end{tabular}

\section{Le sexe}

Les hommes possèdent une attitude plus positive que les femmes. En effet, on peut observer, au tableau 6, que $45.8 \%$ d'entre eux manifestent une attitude plutôt positive comparativement à $25.5 \%$ pour les femmes. D'autre part, les incertains se répartissent de façon quasi égale entre hommes et femmes, avec respectivement $30.4 \%$ et $34.9 \%$.

\section{L'âge}

En mettant en relation les attitudes et l'âge, on observe (tableau 7) que les sujets âgés de 40 à 49 ans possèdent une attitude plus positive (43.6\%) que les autres groupes d'âge ; en effet, les plus jeunes (20-29 ans) sont les moins nombreux à manifester une attitude positive $(23.8 \%)$, suivis des plus âgés ( 50 ans et plus) ( $26.5 \%$ ). On constate également que, à l'exception des 50 ans et plus, il y a dans chacune des catégories d'âge une proportion à peu près équivalente d'intervenants qui ont une attitude négative et incertaine. Finalement, le groupe des $30-39$ ans est le seul qui présente des pourcentages très proches dans less trois blocs d'attitudes. 


\section{TABLEAU 6}

Attitudes selon le sexe.

\begin{tabular}{|c|c|c|c|c|}
\hline Sexe Attitudes & $\begin{array}{l}\text { Plutôt } \\
\text { négative } \\
\% \quad \text { (N) }\end{array}$ & $\begin{array}{l}\text { Incertaine } \\
\% \quad(\mathbf{N})\end{array}$ & $\begin{array}{l}\text { Plutôt } \\
\text { positive } \\
\% \quad \text { (N) }\end{array}$ & $\begin{array}{l}\text { TOTAL } \\
\% \quad(\mathbf{N})\end{array}$ \\
\hline Masculin & $\begin{array}{l}23.8 \quad(88) \\
10.0\end{array}$ & $\begin{array}{l}30.4(112) \\
12.7\end{array}$ & $\begin{array}{l}45.8(169) \\
19.2\end{array}$ & $\begin{array}{r}100.0(369) \\
41.8\end{array}$ \\
\hline Féminin & $\begin{array}{l}39.6(203) \\
23.0\end{array}$ & $\begin{array}{l}34.9(179) \\
20.3\end{array}$ & $\begin{array}{l}25.5(131) \\
14.9\end{array}$ & $\begin{aligned} 100.0(513) \\
58.2\end{aligned}$ \\
\hline TOTAL & $33.0(291)$ & 33.0 (291) & $34.0(300)$ & 100.0 \\
\hline $\begin{array}{c}X^{2}=43.33095 \\
C=.22\end{array}$ & \multicolumn{4}{|c|}{$\begin{array}{c}P=0.0000(<.0001 \text { significatif }) \\
D L=2\end{array}$} \\
\hline
\end{tabular}

\section{TABLEAU 7}

\section{Attitudes selon l'âge.}

\begin{tabular}{|c|c|c|c|c|}
\hline Âge Attitudes & $\begin{array}{l}\text { Plutôt } \\
\text { négative } \\
\% \quad \text { (N) }\end{array}$ & $\begin{array}{l}\text { Incertaine } \\
\begin{array}{c}\% \quad(\mathbf{N})\end{array}\end{array}$ & $\begin{array}{l}\text { Plutôt } \\
\text { positive } \\
\% \quad \text { (N) }\end{array}$ & $\begin{array}{l}\text { TOTAL } \\
\% \quad \text { (N) }\end{array}$ \\
\hline $20-29$ ans & $\begin{aligned} 37.1 & (56) \\
6.3 & \end{aligned}$ & $\begin{aligned} 39.1 & (59) \\
6.7 & \end{aligned}$ & $\begin{aligned} 23.8 & (36) \\
4.1 & \end{aligned}$ & $\begin{array}{c}100.0(151) \\
17.1\end{array}$ \\
\hline $30-39$ ans & $\begin{array}{l}33.2(153) \\
17.3\end{array}$ & $\begin{array}{l}32.1(148) \\
16.8\end{array}$ & $\begin{array}{l}34.7(160) \\
18.1\end{array}$ & $\begin{aligned} 100.0 \\
52.2\end{aligned}$ \\
\hline $40-49$ ans & $\begin{aligned} 28.7 & (54) \\
6.1 & \end{aligned}$ & $\begin{aligned} 27.7 & (52) \\
5.9 & \end{aligned}$ & $\begin{array}{rr}43.6 & (82) \\
9.3 & \end{array}$ & $\begin{array}{c}100.0(188) \\
21.3\end{array}$ \\
\hline 50 ans et plus & $\begin{array}{rr}33.7 & (28) \\
3.2 & \end{array}$ & $\begin{array}{rr}39.8 & (33) \\
3.7 & \end{array}$ & $\begin{aligned} 26.5 & (22) \\
2.5 & \end{aligned}$ & $\begin{aligned} 100.0 & (83) \\
9.4 & \end{aligned}$ \\
\hline TOTAL & $33.0(291)$ & $33.1(292)$ & $34.0(300)$ & $100.0(883)$ \\
\hline $\begin{array}{c}X^{2}=17.53322 \\
C=0.13\end{array}$ & \multicolumn{4}{|c|}{$\begin{array}{c}P=0.0075(<.01 \text { significatif }) \\
D L=6\end{array}$} \\
\hline
\end{tabular}




\section{La discipline d'études}

Par ailleurs, on peut remarquer, au tableau 8 , que seulement $29.3 \%$ des répondants formés en service social démontrent une attitude plutôt positive, comparativement à $42.2 \%$ chez les diplômés des autres disciplines. Les répondants diplômés en service social manifestent, dans une plus grande proportion, une attitude plutôt négative $(36.9 \%)$ qu'incertaine (33.8\%) ou positive $(29.3 \%)$. Chez les diplômés des autres disciplines, cette distribution est inversée : les pourcentages sont de plus en plus élevés $(26.2 \%, 31.6 \%$ et $42.2 \%)$ à mesure qu'on évolue vers des attitudes plus positives.

\section{TABLEAU 8}

Attitudes selon la discipline d'études.

\begin{tabular}{|c|c|c|c|c|}
\hline Discipline & $\begin{array}{l}\text { Plutôt } \\
\text { négative } \\
\% \quad \text { (N) }\end{array}$ & $\begin{array}{l}\text { Incertaine } \\
\% \quad \text { (N) }\end{array}$ & $\begin{array}{l}\text { Plutôt } \\
\text { positive } \\
\% \quad \text { (N) }\end{array}$ & $\begin{array}{l}\text { TOTAL } \\
\% \quad(\mathbf{N})\end{array}$ \\
\hline Service social & $\begin{array}{l}36.9(157) \\
22.4\end{array}$ & $\begin{array}{l}33.8(144) \\
20.5\end{array}$ & $\begin{array}{l}29.3(125) \\
17.8\end{array}$ & $\begin{array}{c}100.0(426) \\
60.8\end{array}$ \\
\hline Autres & $\begin{array}{l}26.2 \quad(72) \\
10.3\end{array}$ & $\begin{array}{l}31.6 \quad(87) \\
12.4\end{array}$ & $\begin{array}{l}42.2(116) \\
16.5\end{array}$ & $\begin{array}{r}100.0 \\
39.2\end{array}$ \\
\hline TOTAL & 32.7 (229) & $33.0(231)$ & 34.4 (241) & $100.0(701)$ \\
\hline $\begin{array}{c}X^{2}=14.07808 \\
C=0.14\end{array}$ & \multicolumn{4}{|c|}{$\begin{array}{c}P=0.0009(<.001 \text { significatif }) \\
D L=2\end{array}$} \\
\hline
\end{tabular}

\section{Le secteur d'activités}

Finalement, en ce qui concerne les secteurs d'activités, on observe que les intervenants ouvrant dans l'administration manifestent une attitude plus positive que ceux des autres secteurs. Le tableau 9 montre que les membres des services administratifs ont, à $73.7 \%$, une attitude plutôt positive alors que dans les autres secteurs, les proportions oscillent entre $27.8 \%$ et $29.7 \%$. 


\section{TABLEAU 9}

Attitudes selon le secteur d'activités.

\begin{tabular}{|c|c|c|c|c|}
\hline Secteur & $\begin{array}{l}\text { Plutôt } \\
\text { négative } \\
\% \quad \text { (N) }\end{array}$ & $\begin{array}{l}\text { Incertaine } \\
\qquad \quad \text { (N) }\end{array}$ & $\begin{array}{l}\text { Plutôt } \\
\text { positive } \\
\% \quad \text { (N) }\end{array}$ & $\begin{array}{l}\text { TOTAL } \\
\% \quad(\mathbf{N})\end{array}$ \\
\hline $\begin{array}{l}\text { Services jeunesse et } \\
\text { famille }\end{array}$ & $\begin{array}{l}35.6(151) \\
17.6\end{array}$ & $\begin{array}{l}36.6(155) \\
18.1\end{array}$ & $\begin{array}{l}27.8(118) \\
13.8\end{array}$ & $\begin{aligned} & 100.0(424) \\
& 49.5\end{aligned}$ \\
\hline $\begin{array}{l}\text { Services aux adultes, } \\
\text { personnes âgées, } \\
\text { handicapées, ser- } \\
\text { vices de santé }\end{array}$ & $\begin{array}{l}41.2 \quad(93) \\
10.9\end{array}$ & $\begin{aligned} 30.1 & (68) \\
7.9 & \end{aligned}$ & $\begin{array}{rr}28.8 & (65) \\
7.6 & \end{array}$ & $\begin{array}{c}100.0(226) \\
26.4\end{array}$ \\
\hline $\begin{array}{l}\text { Services adminis- } \\
\text { tratifs }\end{array}$ & $\begin{array}{ll}5.3 & (5) \\
0.6 & \end{array}$ & $\begin{array}{rr}21.1 & (20) \\
2.3 & \end{array}$ & $\begin{aligned} 73.7 & (70) \\
8.2 & \end{aligned}$ & $\begin{array}{r}100.0 \quad(95) \\
11.1\end{array}$ \\
\hline $\begin{array}{l}\text { Services généraux, } \\
\text { ressources }\end{array}$ & $\begin{array}{r}35.1 \quad(39) \\
4.6\end{array}$ & $\begin{array}{r}35.1 \quad(39) \\
4.6\end{array}$ & $\begin{array}{rr}29.7 & (33) \\
3.9 & \end{array}$ & $\begin{array}{l}100.0(111) \\
13.0\end{array}$ \\
\hline TOTAL & $33.6(288)$ & $32.9(282)$ & $33.4(286)$ & $100.0(856)$ \\
\hline $\begin{array}{c}X^{2}=85.55139 \\
C=0.30\end{array}$ & \multicolumn{4}{|c|}{$\begin{array}{c}P=0.0000(<.0001 \text { significatif }) \\
D L=6\end{array}$} \\
\hline
\end{tabular}

\section{Ce qui fait le plus changer les attitudes}

Pour connaître, parmi les variables indépendantes formulées dans les hypothèses et les variables de contrôle, celles qui étaient les plus étroitement liées aux attitudes, nous avons utilisé, à titre exploratoire, la régression simple et la régression multiple. Cette méthode d'analyse permet de saisir jusqu'à quel point on peut expliquer les attitudes à partir d'une ou de plusieurs variables indépendantes. Le coefficient de détermination $\left(\mathrm{R}^{2}\right)$ permet d'estimer la contribution de chacune des variables à la variance des attitudes.

Le tableau 10 nous présente, par ordre d'importance, les variables contribuant de façon significative à la variance des attitudes. Nous pouvons observer que la position hiérarchique est au premier rang et explique $18 \%$ de la variance des attitudes. Viennent aux deuxième et troisième rangs le degré de contact avec l'informatique (12\%) et les secteurs d'activités $(10 \%)$. Bien que les contributions des trois dernières variables soient significatives (sexe, diplôme et discipline d'études), elles demeurent néanmoins négligeables (moins de $5 \%$ ). 
Des onze variables de départ, six contribuent de façon significative à la variance des attitudes. Cinq sont non significatives : la taille de l'établissement, la durée d'informatisation, le type d'établissement, l'âge et les années de travail.

TABLEAU 10

\section{Contribution de chacune des variables indépendantes et de chacune des variables de contrôle à la variance des attitudes.}

\begin{tabular}{|l|rrr|}
\hline \multicolumn{1}{|c|}{ Variables } & $\mathbf{R}^{2 *}$ & F & \multicolumn{1}{c|}{$\begin{array}{c}\text { Seuil de } \\
\text { signification }\end{array}$} \\
\hline Position hiérarchique & .18 & 91.45534 & F significatif \\
Degré de contact \\
avec l'informatique & .12 & 116.20062 & F significatif \\
Secteur d'activités & .10 & 30.06284 & F significatif \\
Sexe & .04 & 34.27208 & F significatif \\
Diplôme & .02 & 6.51685 & F significatif \\
Discipline d'études & .01 & 15.43790 & F significatif \\
\hline
\end{tabular}

* $\mathrm{R}^{2}$ : représente la proportion de variance expliquée par la ou les variables indépendantes.

$F$ : est un test statistique qui permet de vérifier la signification statistique du $R^{2}$.

Examinons maintenant l'effet conjoint de certaines variables. À partir des résultats du tableau 10 , nous jugeons opportun de ne retenir que les variables contribuant pour $5 \%$ et plus à la variance des attitudes : la position hiérarchique, le degré de contact avec l'informatique et les secteurs d'activités. Nous pouvons observer, au tableau 11, que ces trois variables prises conjointement expliquent $22 \%$ de la variance des attitudes. En d'autres termes, cela signifie probablement que plusieurs autres facteurs viennent expliquer la majeure partie de la variation des attitudes à l'égard de l'informatique. Ces résultats ne sont guère surprenants lorsqu'on sait que les attitudes font appel aux connaissances et aux sentiments, ce qui peut impliquer l'influence d'un grand nombre d'éléments difficilement perceptibles. 


\section{TABLEAU 11}

\section{Contribution des trois variables, prises conjointement, à la variance des attitudes.}

\begin{tabular}{|c|ccc|}
\hline Variables & $\mathbf{R}^{2}$ & F & $\begin{array}{c}\text { Seuil de } \\
\text { signification .01 }\end{array}$ \\
\hline $\begin{array}{l}\text { - Position hiérarchique } \\
\text { - Degré de contact avec } \\
\text { l'informatique } \\
\text { - Secteur d'activités }\end{array}$ & 22 & 41.62951 & F significatif \\
\hline
\end{tabular}

Les trois hypothèses formulées dans le cadre théorique, à l'effet que les attitudes des intervenants sociaux varient selon leur position hiérarchique, leur degré de contact avec l'informatique et le type d'établissement, se voient donc confirmées. Les résultats ont démontré que les intervenants en position hiérarchique élevée ou ayant un degré de contact élevé avec l'informatique manifestent une attitude plus positive. On a vu également que les intervenants exerçant leurs fonctions dans un C.S.S. ont une attitude plus favorable que ceux œuvrant dans les C.L.S.C. Ces résultats rejoignent les observations relevées dans la littérature. En effet, dans son étude, Lynch (1985) démontre que les administrateurs présentent des attitudes plus positives à l'égard de l'informatique que les praticiens.

Par ailleurs, les deux hypothèses à l'effet que les attitudes varient selon la taille de l'établissement et la durée d'informatisation ne se sont pas vérifiées. Nos résultats ne concordent pas avec ce qu'ont observé Pinkerton et Raffoul (1984), qui croient que les attitudes à l'égard de l'informatique varient selon la taille (petite ou grande) de l'organisation. Comme ces auteurs utilisent des nombres d'employés différents pour définir la taille de l'organisation, il est possible que l'écart dans les résultats s'explique par le mode d'opérationnalisation du concept " taille de l'organisation".

Dans un autre ordre d'idées, les données non significatives quant à la durée d'informatisation nous laissent croire qu'il est peut-être trop tôt pour espérer voir apparaître des différences dans les attitudes à ce niveau, surtout lorsqu'on considère que l'ensemble des établissements (qu'ils soient informatisés depuis peu ou plusieurs années), ne sont pas très avancés dans le développement de l'informatisation. Le degré de développement peut être très faible, voire même négligeable dans 
certains cas si on se replace dans le contexte de la période où la cueillette des données s'est effectuée (automne 1986).

En somme, les attitudes à l'égard de l'informatique semblent fluctuer davantage à partir des caractéristiques personnelles des intervenants qu'à partir de caractéristiques de type organisationnel. En effet, nous avons pu constater que les attitudes varient selon le sexe, l'âge, la discipline d'études et le secteur d'activités, facteurs relevant des individus et non du contexte organisationnel.

Quoique, dans l'ensemble, les intervenants semblent avoir une attitude plutôt favorable à l'égard de l'informatique dans leur travail, nous avons vu que leurs opinions divergent considérablement selon leur position hiérarchique ou leur degré de contact avec l'informatique.

\section{Conclusion}

Nous avons examiné les relations entre les attitudes et les variables indépendantes comprises dans les hypothèses, et les variables de contrôle. Parmi ces variables, plusieurs se sont avérées en relation significative avec les attitudes: la position hiérarchique, le degré de contact avec l'informatique, le type d'établissement, le sexe, l'âge, la discipline d'études et le secteur d'activités. Les résultats nous ont permis de découvrir que ceux qui ont une attitude positive sont:

- les intervenants dans une position hiérarchique élevée ;

- les intervenants ayant un degré de contact élevé avec l'informatique ;

- les intervenants travaillant dans les C.S.S. plus que ceux œuvrant dans les C.L.S.C.;

- les hommes plus que les femmes;

- les intervenants âgés de 40 à 49 ans plus que les autres catégories d'âge ;

- les intervenants diplômés en sciences humaines plus que ceux formés en service social ;

- les intervenants ouvrant dans les services administratifs plus que ceux des autres secteurs.

Par ailleurs, les analyses de régression nous ont permis d'explorer les liens entre les variables et les attitudes à l'égard de l'informatique. Parmi les variables significatives, la position hiérarchique est celle qui explique la plus grande proportion de variabilité dans les attitudes $(18 \%)$. Les variables position hiérarchique, degré de contact avec l'informatique et secteur d'activités, prises conjointement, expliquent $22 \%$ de la variance des attitudes, soit $4 \%$ seulement de plus que la position hiérarchique à elle seule. 
Comme on le voit, il reste beaucoup à découvrir pour arriver à interpréter ou à prédire les attitudes à l'égard de l'informatique. Les résultats reflètent un niveau important d'ambivalence dans les attitudes des répondants. Seulement deux des vingt-trois énoncés de l'échelle d'attitudes ont moins de $20 \%$ d'incertains, alors que les autres dépassent $20 \%$ pour atteindre un niveau supérieur à $40 \%$ dans deux cas.

Ces résultats vont dans le même sens que ceux observés dans d'autres recherches menées ailleurs au Canada et aux États-Unis. Le processus d'informatisation, commencé depuis plusieurs années dans le réseau des services sociaux québécois, va se poursuivre et même s'accentuer. Il importe donc que ce processus soit accompagné par la recherche afin d'apporter l'éclairage nécessaire à sa réussite et à son évaluation. Comme il paraît de plus en plus évident que les nouveaux développements vont s'orienter vers les applications reliées à la pratique professionnelle, il est d'autant plus nécessaire qu'ils soient suivis de façon plus systématique encore que par le passé, en raison des implications plus directes pour la clientèle.

\section{Note}

1 Le rapport complet de recherche est disponible au Laboratoire de recherche de l'École de service social de l'Université Laval sous le titre : Les perceptions et les attitudes des intervenants sociaux du Québec au sujet de l'utilisation de l'informatique dans leur milieu de travail, Laboratoire de recherche, École de service social, Université Laval, juillet 1987, 204p.

\section{Références bibliographiques}

Bergeron, J. et al., Les aspects humains de l'organisation, Chicoutimi, Gaëtan Morin, 1979.

GUMMER, B., "A byte of the Apple : Current perspectives on information technology ", Administration in Social Work, vol. 10, no 4, $1986: 81-94$.

LYNCH, D., The Computerization of Information Systems in Community Mental Health Centers, thèse de doctorat en service social, Chicago (III.), Université de Chicago, 1985.

Pinkerton, G.L. et P.R. Raffoul, "Professional colleagues: Confronting the attitudes of professionals toward microcomputers ", dans: M.D. SCHWARTZ (éd.), Using Computers in Clinical Practice, New York, The Haworth Press, 1984 : 61-66. 